Louisiana State University

LSU Digital Commons

Faculty Publications

Department of Physics \& Astronomy

2-1-2014

\title{
The galactic bulge survey: Completion of the X-ray survey observations
}

\author{
Peter G. Jonker \\ SRON Netherlands Institute for Space Research \\ Manuel A.P. Torres \\ SRON Netherlands Institute for Space Research \\ Robert I. Hynes \\ Louisiana State University \\ Thomas J. Maccarone \\ Texas Tech University \\ Danny Steeghs \\ University of Warwick
}

See next page for additional authors

Follow this and additional works at: https://digitalcommons.Isu.edu/physics_astronomy_pubs

\section{Recommended Citation}

Jonker, P., Torres, M., Hynes, R., Maccarone, T., Steeghs, D., Greiss, S., Britt, C., Wu, J., Johnson, C., Nelemans, G., \& Heinke, C. (2014). The galactic bulge survey: Completion of the X-ray survey observations. Astrophysical Journal, Supplement Series, 210 (2) https://doi.org/10.1088/0067-0049/210/ $2 / 18$

This Article is brought to you for free and open access by the Department of Physics \& Astronomy at LSU Digital Commons. It has been accepted for inclusion in Faculty Publications by an authorized administrator of LSU Digital Commons. For more information, please contact ir@lsu.edu. 


\section{Authors}

Peter G. Jonker, Manuel A.P. Torres, Robert I. Hynes, Thomas J. Maccarone, Danny Steeghs, Sandra Greiss, Christopher T. Britt, Jianfeng Wu, Christopher B. Johnson, Gijs Nelemans, and Craig Heinke 


\title{
THE GALACTIC BULGE SURVEY: COMPLETION OF THE X-RAY SURVEY OBSERVATIONS
}

\author{
Peter G. Jonker ${ }^{1,2,3}$, Manuel A. P. Torres ${ }^{1,3}$, Robert I. Hynes ${ }^{4}$, Thomas J. Maccarone ${ }^{5}$, \\ Danny Steeghs ${ }^{6}$, Sandra Greiss ${ }^{6}$, Christopher T. Britt ${ }^{4,5}$, Jianfeng Wu ${ }^{3}$, Christopher B. Johnson ${ }^{4}$, \\ Gijs Nelemans $^{2,7}$, AND CRaig Heinke 8 \\ ${ }^{1}$ SRON, Netherlands Institute for Space Research, Sorbonnelaan 2, 3584-CA, Utrecht, The Netherlands \\ ${ }^{2}$ Department of Astrophysics/IMAPP, Radboud University Nijmegen, Heyendaalseweg 135, 6525-AJ, Nijmegen, The Netherlands \\ ${ }^{3}$ Harvard-Smithsonian Center for Astrophysics, 60 Garden Street, Cambridge, MA 02138, USA \\ ${ }^{4}$ Department of Physics and Astronomy, Louisiana State University, Baton Rouge, LA 70803-4001, USA \\ ${ }^{5}$ Department of Physics, Texas Tech University, P.O. Box 41051, Lubbock, TX 79409-1051, USA \\ ${ }^{6}$ Astronomy and Astrophysics, Department of Physics, University of Warwick, Coventry CV4 7AL, UK \\ ${ }^{7}$ Institute for Astronomy, KU Leuven, Celestijnenlaan 200D, B-3001, Leuven, Belgium \\ ${ }^{8}$ Department of Physics, University of Alberta, Room 238 CEB, Edmonton AB T6G 2G7, Canada \\ Received 2013 December 2; accepted 2013 December 11; published 2014 January 10
}

\begin{abstract}
We provide the Chandra source list for the last $\sim$ quarter of the area covered by the Galactic Bulge Survey (GBS). The GBS targets two strips of $6^{\circ} \times 1^{\circ}\left(12\right.$ square degrees in total), one above $\left(1^{\circ}<b<2^{\circ}\right)$ and one below $\left(-2^{\circ}<b<-1^{\circ}\right)$ the Galactic plane in the direction of the Galactic center at X-ray, optical, and near-infrared wavelengths. For the X-ray part of the survey we use $2 \mathrm{ks}$ per Chandra pointing. We find $424 \mathrm{X}$-ray sources in the 63 Chandra observations on which we report here. These sources are in addition to the $1216 \mathrm{X}$-ray sources discovered in the first part of the GBS survey described previously. We discuss the characteristics and the X-ray variability of the brightest of the sources as well as the radio properties from existing radio surveys. We point out an interesting asymmetry in the number of X-ray sources as a function of their Galactic $l$ and $b$ coordinates which is probably caused by differences in average extinction toward the different parts of the GBS survey area.
\end{abstract}

Key words: accretion, accretion disks - binaries: symbiotic - novae, cataclysmic variables - stars: activity $\mathrm{X}$-rays: binaries

Online-only material: color figures, machine-readable table

\section{INTRODUCTION}

Stellar mass black holes and neutron stars are the end points of massive star evolution via supernovae or gamma-ray bursts. Nearly all of the Galactic black holes and many of the neutron stars found so far are located in binaries. Their properties are the observable consequences of binary interactions. Studying these remnants provides vital clues to understanding the evolutionary processes that produce them both in terms of single massive star evolution and binary star evolution. For example, the current stellar mass black hole distribution based on a sample of about 20 objects appears to be disjoint from that of neutron stars (Özel et al. 2010, 2012; Farr et al. 2011) suggesting a bimodality in formation that produces either low-mass neutron stars or relatively high-mass black holes, with few systems in between. This remains a challenge for supernova models to reproduce (Fryer et al. 2012; Belczynski et al. 2012). Kreidberg et al. (2012) argue that this mass gap may be due, in part, to systematic effects underestimating the system inclination.

Unfortunately, our observational sample, particularly in the case of black holes, is largely comprised of objects discovered in transient X-ray outbursts, leading to a variety of possible selection effects that could obscure the properties of the true population (e.g., see Narayan \& McClintock 2005). For instance, using the disk instability model including disk irradiation effects (see Lasota 2008), one could envisage an inverse correlation between the accretor mass and the duty cycle, reducing the chance of detection of relatively low-mass black holes in outbursts. Additional selection effects could be invoked by the black-hole-mass-orbital-period correlation (Lee et al. 2002) and, possibly related to that, the optical and X-ray outburst peak-luminosity-orbital-period correlation (Shahbaz \& Kuulkers 1998 and Wu et al. 2010, respectively).

To mitigate the selection effects incurred by selecting systems that recently went through an outburst cycle we designed the Galactic Bulge Survey (GBS; Jonker et al. 2011). The GBS is a wide, shallow Chandra X-ray survey of the Galactic Bulge aiming to uncover many $(>100)$ new quiescent black hole and neutron star binaries. As a result, we may find sources quite different from those identified in outbursts. A second goal of the survey is to constrain binary evolution models (e.g., King \& Ritter 1999; Pfahl et al. 2002; Belczynski \& Taam 2004) using the observed number ratio between the $\approx 100 \mathrm{X}$-ray binaries and several hundred CVs that we expect to find. This number will particularly put constraints on uncertain phases in the binary evolution such as the common envelope phase (e.g., Kiel \& Hurley 2006; Ivanova et al. 2013).

For both these science goals we need to classify the X-ray sources. Given that this classification relies on multi-wavelength data, by design, the survey area is sufficiently out of the plane to allow (multi-epoch) optical and near-infrared (NIR) follow-up of the majority of detected sources. In addition to classification, optical and NIR spectroscopic observations are also crucial for dynamical studies to derive compact object masses (and sometimes the dynamical masses are necessary for classification, e.g., Ratti et al. 2013).

The GBS is well under way. Radio counterparts of a sample of sources from the first part of the X-ray survey have been identified by Maccarone et al. (2012). Hynes et al. (2012) reported on associations of X-ray sources with the brightest optical counterparts. Results from optical variability alone (Udalski et al. 2012) and optical variability and spectroscopic 
studies together (Ratti et al. 2013; Britt et al. 2013; Hynes et al. 2014; Torres et al. 2013) are appearing. Furthermore, we are using NIR observations from the Two Micron All Sky Survey (2MASS), VISTA Variables in The Via Lactea (VVV), and the UKIRT Infrared Deep Sky Survey to identify counterparts of the GBS X-ray sources (Greiss et al. 2014).

Here we report on Chandra observations of the final $\approx$ quarter of the sky area of 12 square degrees that makes up the GBS, completing the Chandra survey observations of the GBS area. The initial three quarters were reported in Jonker et al. (2011). In addition, we provide the radio counterparts to the X-ray sources discovered in the final part after Maccarone et al. (2012) reported on archival radio sources for the first three quarters. Finally, we investigate the spatial distribution of all the X-ray sources found in the GBS area, and by comparing these with the ROSAT sources in the sky area on which we report here, we investigate the variability properties of the new GBS X-ray sources.

\section{CHANDRA X-RAY OBSERVATIONS, ANALYSIS, AND RESULTS}

\subsection{Source Detection}

We have obtained 63 observations with the Chandra X-Ray Observatory (Weisskopf et al. 2002) covering the remaining quarter of the total area of 12 square degrees that we call the GBS. We employed the same analysis tools and techniques as described in Jonker et al. (2011) as much as possible in order to come to as homogeneous a survey as possible. Also we follow the source naming convention introduced there, where sources reported in Jonker et al. (2011) are referred to as CX\# (after Chandra X-ray source, where the numeral indicates the position of that source in the list, with sources providing the largest number of counts at detection having the lowest numeral), while new sources found in the 63 new observations are called CXB\#.

In the left panel in Figure 1 we show the 63 new Chandra observations reported on here. The red curved line indicates the composite outline of each circular field of view of $14^{\prime}$ diameter for these 63 observations. The gray curved lines bordering the white points indicate the composite outline of each circular field of view of $14^{\prime}$ diameter of the individual Chandra observations obtained and the detected sources reported in Jonker et al. (2011), respectively. The area near $l=0^{\circ}$ is covered by the observations from Hong et al. (2009). Sources found in $2 \mathrm{ks}$ segments of those exposures were listed in Jonker et al. (2011) as well. In the right panel in Figure 1 the white circles indicate the position of the detected point sources. The size of the white circles is an indication of the number of Chandra counts detected for that particular source.

The Chandra observations have been performed using the I0-I3 CCDs of the Advanced CCD Imaging Spectrometer (ACIS) detector (Garmire 1997; ACIS-I). The observation identification (ID) numbers for the data presented here are 13528-13590. We reprocessed and analyzed the data using the CIAO 4.3 software developed by the Chandra X-ray Center and employing CALDB version 4.4.6. The data telemetry mode was set to very faint for all observations. The very faint mode provides $5 \times 5$ pixel information per X-ray event. This allows for a better screening of events caused by cosmic rays. In our analysis we selected events only if their energy falls in the $0.3-8 \mathrm{keV}$ range.

We usedWAVDETECT to search for X-ray sources in each of the observations using data covering the full $0.3-8$, the
$0.3-2.5$, and the 2.5-8 keV energy bands, separately. We set the SIGTHRESH in WAVDETECT to $1 \times 10^{-7}$, which implies that for a background count rate constant over the ACIS-I CCDs there would be $<0.1$ spurious source detection per observation as about $1 \times 10^{6}$ pixels are searched per observation. However, in most cases a source is not detected in a single pixel, thus our estimate of 0.1 spurious source per observation is very conservative. Furthermore, as we explain below, we applied additional selection criteria. This further lowers the number of spurious sources.

We retained all sources for which Poisson statistics indicate that the probability of obtaining the number of detected source counts by chance, given the expectation for the local background count rate, is lower than $1 \times 10^{-6}$. This would be equivalent to a $>5 \sigma$ source detection in Gaussian statistics. Next, we deleted all sources for which WAVDETECT was not able to provide an estimate of the uncertainty on the right ascension $(\alpha)$ and on declination $(\delta)$ as this often indicates that all counts fell in 1 pixel which could well be due to faint afterglow events caused by cosmic-ray hits. In addition, we impose a three count minimum for source detection as Murray et al. (2005) simulated that in their XBootes survey with $5 \mathrm{ks}$ ACIS-I exposures, $14 \%$ of the two-count sources were spurious (note that this percentage will probably be lower for our GBS exposures of $2 \mathrm{ks}$ ).

Since our Chandra observations were designed to overlap near the edges, we searched for multiple detections of the same source either in one of the energy sub-bands or in the full energy band. We consider sources with positions falling within $5^{\prime \prime}$ of each other likely multiple detections of the same source. This radius is larger than that of $3^{\prime \prime}$ which we took in Jonker et al. (2011) as we found out that some multiple detections of the same source still remained for sources detected with large offaxis angles (see Hynes et al. 2012 for the list of 18 sources from Jonker et al. 2011 that were in fact multiple detections of the same source.) This means that in Jonker et al. (2011) we found 1216 unique sources.

In the last quarter of the GBS area that we report on here, we found that 26 sources are detected more than once. Out of these 26 sources, 23 sources are detected two times, and 3 sources are detected three times. Two of the sources detected twice were already detected and reported in Jonker et al. (2011; CX155 and CX314). We do not list these two sources in Table 1. The properties that we list in Table 1 for the sources that are detected multiple times are those for the detection that gave rise to the largest number of X-ray counts. In Table 1 we also list the number of times that sources are detected.

Besides the multiple detections of CX155 and CX314, 14 additional sources detected once in the cycle 13 Chandra observations were previously detected and listed in Jonker et al. (2011). These sources are CX15, CX17, CX25, CX44, CX60, CX69, CX79, CX137, CX221, CX266, CX312, CX355, CX374, and CX439. In most cases the off-axis angle of the source position was larger during the new observations and, given that a similar number of X-ray counts was detected in each instance, the source position provided in Jonker et al. (2011) is the most accurate X-ray position available. The main exception where we consider the newly derived position to be more accurate is CX314. CX314 was detected at 10.8 off-axis at eight counts in the Chandra detection leading to its discovery. The new detection we report on here provides 17 counts and the source was 5'9 off-axis in ObsID 13581. The new best-fit source position is $(\alpha, \delta)=(266.6461515,-31.8136964)$ which is 2 . 6 from the previously reported position. 


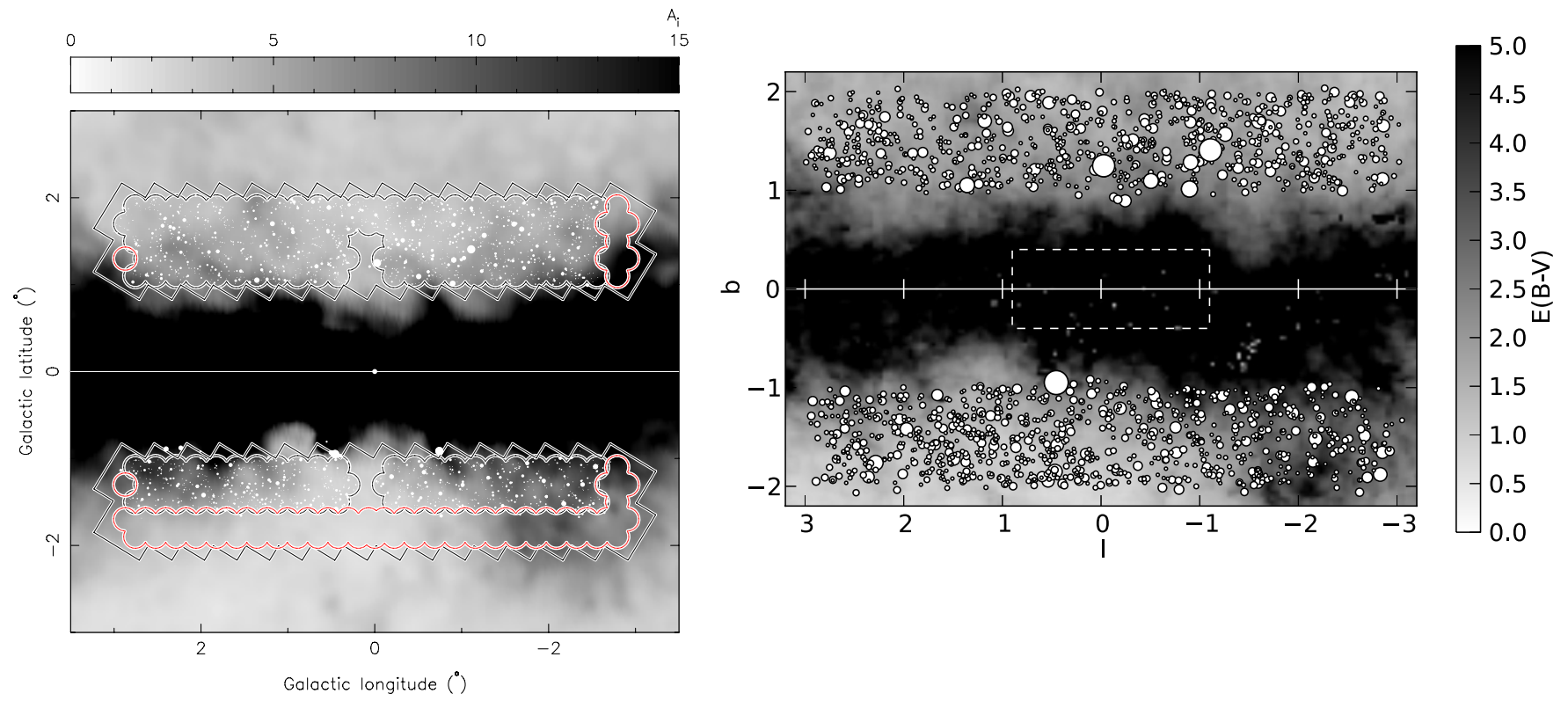

Figure 1. Left panel: the large black, white-rimmed saw-tooth boxes are the outline of our optical observations of the GBS area in Galactic coordinates. The gray-scale image depicts the total reddening in the Sloan $i^{\prime}$-band filter, $A_{i^{\prime}}$, estimated from the COBE dust maps (Schlegel et al. 1998). The overplotted white circles indicate the position of the Chandra X-ray sources detected in the GBS reported in Jonker et al. (2011). The sources found in the areas near $l=0^{\circ}$ and $1^{\circ}<|b|<2^{\circ}$ were reported in Jonker et al. (2011) but the observations were from Hong et al. (2009). The red-rimmed curved lines indicate the composite outline of each circular field of view of 14' diameter for the 63 Chandra observations that we report on in this paper. Right panel: the gray-scale image and contours depict the total absorption $E(B-V)$, estimated from the extinction maps from the VVV (Gonzalez et al. 2012). The overplotted white circles indicate the position of all X-ray sources detected in the GBS including the new sources reported on here. The size of the white circles is proportional to the number of Chandra counts detected for that particular source. The dashed rectangle outlines the region of the survey of the Galactic center from Wang et al. (2002).

Table 1

The GBS X-Ray Source List Providing the GBS Source Name

\begin{tabular}{|c|c|c|c|c|c|c|c|c|c|c|c|}
\hline $\begin{array}{l}\text { Source } \\
\text { Name }\end{array}$ & CXB\# & $\begin{array}{c}\alpha \\
\text { (degrees) }\end{array}$ & $\begin{array}{c}\delta \\
\text { (degrees) }\end{array}$ & $\begin{array}{l}\Delta \alpha \\
\left({ }^{\prime \prime}\right)\end{array}$ & $\begin{array}{l}\Delta \delta \\
(")\end{array}$ & $\begin{array}{c}\# \\
\text { (cnt) }\end{array}$ & Obs ID & $\begin{array}{c}\text { Off-axis Angle } \\
\left({ }^{\prime}\right)\end{array}$ & $\begin{array}{c}\text { \# of } \\
\text { Detec. }\end{array}$ & $\begin{array}{c}\Delta \text { pos } \\
\left({ }^{\prime \prime}\right)\end{array}$ & $\mathrm{HR}$ \\
\hline CXOGBSJ175748.7-275214 & CXB1 & 269.4529160 & -27.8707194 & 0.19 & 0.22 & 161 & 13536 & 7.74 & 1 & 0.74 & $-0.61 \pm 0.06$ \\
\hline CXOGBSJ175359.8-292907 & CXB2 & 268.4994759 & -29.4852781 & 0.09 & 0.05 & 148 & 13550 & 4.35 & 2 & 0.35 & $-0.18 \pm 0.02$ \\
\hline CXOGBSJ174614.3-321949 & CXB3 & 266.5599883 & -32.3303786 & 0.06 & 0.05 & 105 & 13574 & 2.64 & 1 & 0.31 & $0.28 \pm 0.03$ \\
\hline CXOGBSJ173416.2-304538 & CXB4 & 263.5678548 & -30.7607505 & 0.15 & 0.09 & 70 & 13586 & 3.78 & 1 & 0.51 & $-0.90 \pm 0.12$ \\
\hline CXOGBSJ173208.6-302828 & CXB5 & 263.0362304 & -30.4746348 & 0.07 & 0.10 & 66 & 13587 & 3.78 & 1 & 0.53 & $-0.75 \pm 0.10$ \\
\hline CXOGBSJ174517.0-321356 & CXB6 & 266.3208565 & -32.2323620 & 0.11 & 0.11 & 66 & 13577 & 3.73 & 2 & 0.52 & $0.78 \pm 0.11$ \\
\hline CXOGBSJ175551.6-283213 & CXB7 & 268.9650346 & -28.5369772 & 0.06 & 0.05 & 65 & 13533 & 1.83 & 1 & 0.32 & $0.34 \pm 0.05$ \\
\hline CXOGBSJ175432.1-292824 & CXB8 & 268.6339299 & -29.4734138 & 0.28 & 0.26 & 65 & 13550 & 7.49 & 2 & 1.42 & $-0.78 \pm 0.11$ \\
\hline CXOGBSJ174916.6-311518 & CXB9 & 267.3192034 & -31.2550666 & 0.09 & 0.07 & 64 & 13569 & 3.52 & 1 & 0.50 & $-0.95 \pm 0.13$ \\
\hline CXOGBSJ175832.4-275244 & CXB10 & 269.6350093 & -27.8789043 & 0.13 & 0.11 & 53 & 13558 & 4.30 & 1 & 0.68 & $-0.56 \pm 0.09$ \\
\hline
\end{tabular}

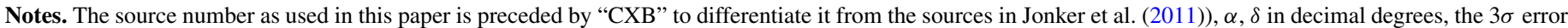

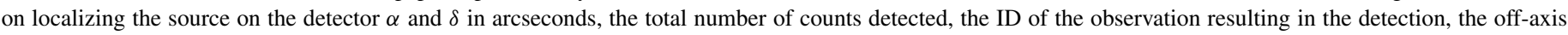

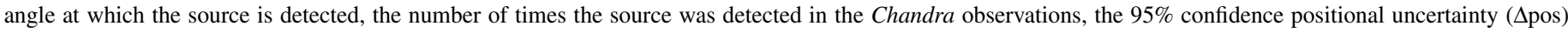

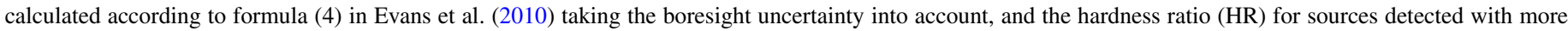

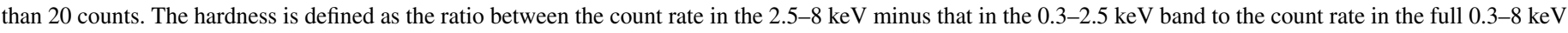
energy band. The HR is calculated for the detection where the off-axis angle was smallest if the source was detected multiple times.

(This table is available in its entirety in a machine-readable form in the online journal. A portion is shown here for guidance regarding its form and content.)

Others, like CX25, were detected closer on-axis in the new cycle 13 observations (6.7 off-axis with six counts) but with many more counts in the observation reported in Jonker et al. (2011; 7'.2 off-axis with 48 counts) than in the new cycle 13 observation implying that the position provided in Jonker et al. (2011) will be more accurate. We do conclude that CX25 is variable in X-rays.

In total we detected 424 distinct sources in the area indicated with red circles and the red curved lines on the left side in Figure 1. The source list is given in Table 1 and the table provides information on $\alpha, \delta$, the error on $\alpha$ and $\delta$, total number of counts detected, the observation ID of the observation resulting in the detection and the off-axis angle at which the source is detected. The errors on $\alpha$ and $\delta$ are the errors provided by WAVDETECT, and do not take into account the typical Chandra boresight uncertainty of $0{ }^{\prime \prime} 6$ (90\% confidence). We do, however, add a column to Table 1 quoting the total uncertainty on the source position following formula (4) in Evans et al. (2010). For clarity, we repeat their equation here:

$$
\log P= \begin{cases}0.1145 \theta-0.4957 & \log C+0.1932 \\ & \text { for } 0.0<\log C<2.1393 \\ 0.0968 \theta-0.2064 & \log C-0.4260 \\ & \text { for } 2.1393<\log C<3.3\end{cases}
$$




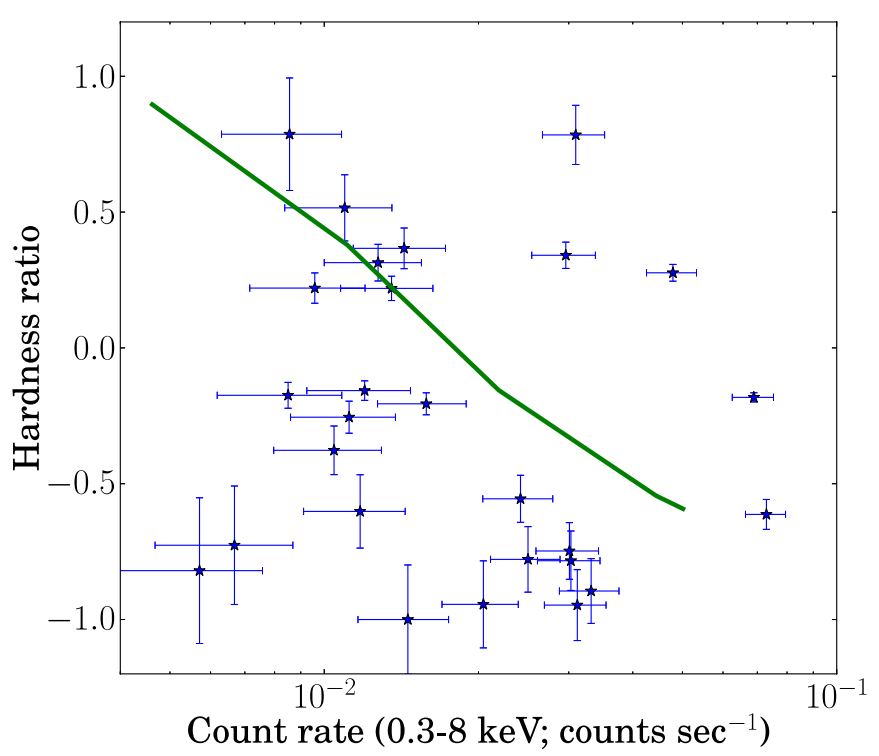

Figure 2. Hardness-intensity diagram for the 27 sources for which 20 or more counts were detected in Chandra cycle 13 observations for the GBS survey. To mitigate effects of small differences in exposure times we used count rates as a measure of intensity. The hardness is defined as the ratio between the count rate in the $2.5-8 \mathrm{keV}$ minus that in the $0.3-2.5 \mathrm{keV}$ band to the count rate in the full $0.3-8 \mathrm{keV}$ energy band. Hard sources fall in the top half and soft sources in the bottom half of this figure. The green line shows the influence of the extinction $\left(N_{\mathrm{H}}\right)$ on a power-law spectrum with index 2 for a source count rate of 0.05 counts $\mathrm{s}^{-1}$ and $N_{\mathrm{H}}$ values increasing from bottom right to top left from $(0.01,0.1,1,3,10) \times 10^{22} \mathrm{~cm}^{-2}$.

(A color version of this figure is available in the online journal.)

where $\theta$ is the off-axis angle in arcminutes and $C$ is the detected number of $\mathrm{X}$-ray photons. The positional error $P$ is given in arcseconds and it corresponds to a $95 \%$ confidence interval.

We provide individual Chandra source names, however, for briefness we use the source number in Table 1 preceded by "CXB" to indicate which source we discuss in this paper. For the error $\sigma_{N}$ on the detected number of counts $N$, Grimm et al. (2005) give $\sigma_{N}=1+\sqrt{N+0.75}$ after Gehrels (1986). To allow for a rough, easy calculation of the source flux based on the detected number of source counts we give the conversion factor for a source spectrum of a power law with photon index of 2 absorbed by $N_{\mathrm{H}}=1 \times 10^{22} \mathrm{~cm}^{-2}: 7.76 \times$ $10^{-15} \mathrm{erg} \mathrm{cm}^{-2} \mathrm{~s}^{-1}$ photon $^{-1}$.

\subsection{X-ray Spectral Information}

We extract source counts using circular source extraction regions of $10^{\prime \prime}$. Background extraction regions are annulli with inner and outer radii of $15^{\prime \prime}$ and $30^{\prime \prime}$, respectively. We plot the 27 sources for which we detected 20 or more counts in a hardness-intensity diagram (Figure 2). To mitigate the effects that small differences in exposure time across our survey can have, we use count rates as a measure of intensity. We define the hardness ratio (HR) as the ratio between the count rate in the $2.5-8 \mathrm{keV}$ minus that in the $0.3-2.5 \mathrm{keV}$ band to the count rate in the full $0.3-8 \mathrm{keV}$ energy band (after Kim et al. 2004). We derived the hardness using XSPEC version 12.7 (Arnaud 1996) by determining the count rates in the soft and hard band taking the response and ancillary response file for each of the sources. For these 27 sources photon pile-up is less than $10 \%$ even for the brightest source. Naively, one would expect most hard sources to be more distant and more absorbed than the soft sources, as the intrinsic spectral shape of the most numerous classes of sources we expect to find does not differ much.

The most interesting aspect from Figure 2 is perhaps the presence of three bright (rate $>2.5 \times 10^{-2}$ counts $\mathrm{s}^{-1}$ ) and relatively hard sources $(\mathrm{HR}>0)$. Their relatively hard spectrum makes it likely that these three sources $(\mathrm{CXB} 3[\mathrm{HR}=0.28 \pm$ $0.03]$, CXB6 [HR $=0.78 \pm 0.11]$, and CXB7 [HR $=0.34 \pm$ 0.05]) suffered significantly from X-ray absorption thus they are likely at a distance of more than $3 \mathrm{kpc}$, which, given their relatively high X-ray flux, means that their X-ray luminosity is substantial. CXB3 is probably a transient source (see below) and none of the three sources is associated with archival radio emission (see below) decreasing the chance that they are background active galactic nuclei (AGNs), and making them potential X-ray binaries.

As foreseen, the spectral information is insufficient for source classification for the majority of the total number of detected sources, therefore, classification will have to come from (multiepoch) multi-wavelength observations. Finally, there seems to be a dichotomy in the hardness with one peak centered on a hardness of 0.2 and another centered on -0.8 with a paucity of sources with hardness 0 . A similar dichotomy was reported in Warwick et al. (2011) and Jonker et al. (2011); see the latter paper for a possible explanation for the nature of this dichotomy.

\subsection{Chandra Light Curves of Source CXB\#1-10}

We inspect the Chandra light curves of source CXB\#1-10. We rebinned the light curves in $200 \mathrm{~s}$ bins. Sources CXB\#1, 2, 3,6 , and 9 show suggestive evidence for flare-like variability. Fitting the light curve with a constant gives a $\chi^{2}$ value of 16 (for 10 degrees of freedom (dof)), 35.9 (9 dof), 19.5 (10 dof), 18 (10 dof), 16.4 (9 dof), respectively. The light curves of source CXB\# 4, 5, 7, 8, and 10 are consistent with being constant with $\chi^{2}$ values of 8.4 (10 dof), 7.5 (9 dof), 11 (10 dof), 10 (9 dof), and 3.8 (10 dof), respectively. We do note that the number of counts in each $200 \mathrm{~s}$ bin varies between 35 and 3 counts between these sources and as a function of time. Therefore, certainly for the bins containing only a few counts the use of the $\chi^{2}$ statistic is suspect. The small number of counts per bin in several cases makes it likely that some of the high values of reduced $\chi^{2}$ are occurring due to chance fluctuations.

In Figure 3 we plot the light curves of the sources for which there is evidence for variability during the observations (i.e., CXB1, CXB2, CXB3, CXB6, and CXB9) and for comparison we plot in the top panel of the same figure the light curve of CXB10 for which our current data provides no evidence that the source varies during the observation.

\section{DISCUSSION}

Using 63 Chandra observations we cover the remaining $\approx$ quarter of the 12 square degrees that comprise the GBS (Jonker et al. 2011). In this paper we provide the list of $424 \mathrm{X}$-ray sources that we find in this area and that have three or more counts in the short (2 ks) Chandra observations.

In total we detected 1640 unique X-ray sources. Of these, 875 are detected at Galactic latitudes below the plane and 765 at Galactic latitudes above the plane. For a symmetric distribution of 1640 sources one would expect $820 \pm 20$ on either side, making the detected distribution marginally skewed. We investigated the nature of this asymmetry by dividing the number of sources over the four quadrants in which they were detected. We made quadrants according to the Galactic coordinates of the 

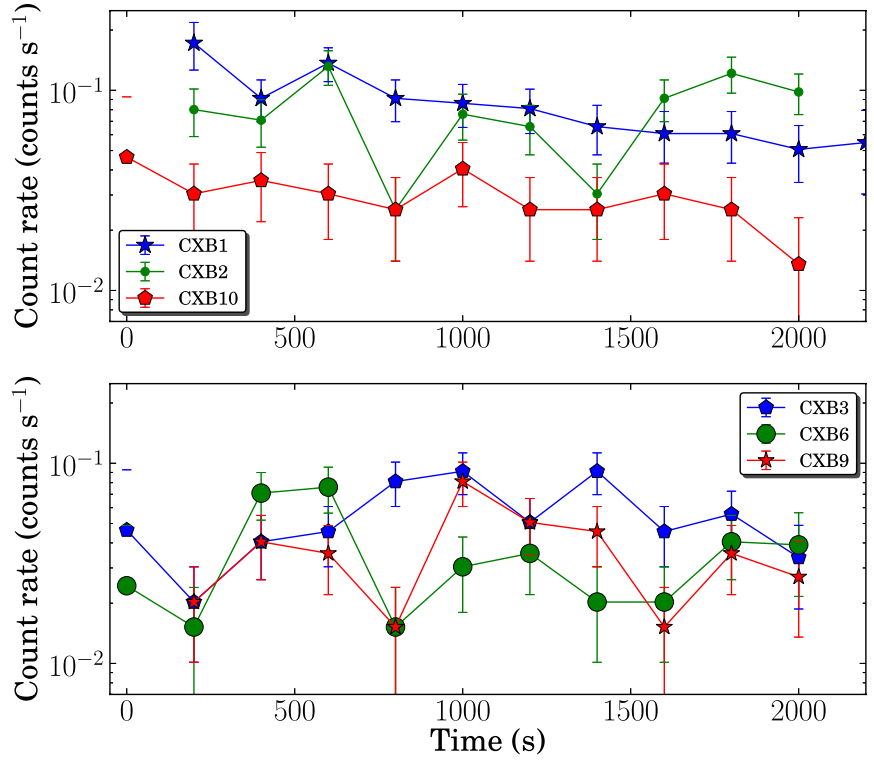

Figure 3. Chandra X-ray light curves of six CXB sources. Each point is an average of $200 \mathrm{~s}$ of Chandra data. For five sources there is suggestive evidence that the source is variable during the Chandra observation (CXB1, CXB2, CXB3, CXB6, and CXB9). For comparison we also plot in the top panel the light curve of CXB10 for which we find no evidence that the source varied during the observation.

(A color version of this figure is available in the online journal.)

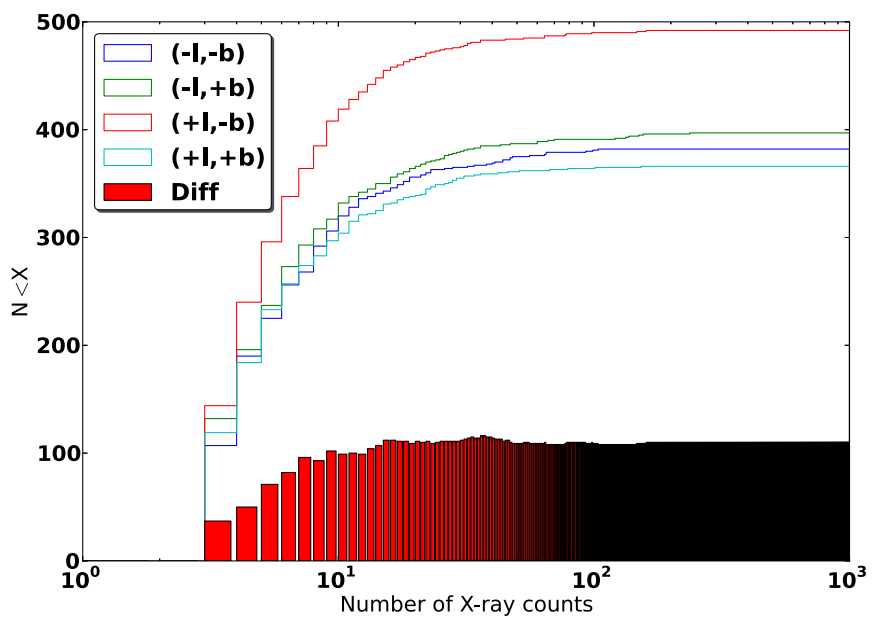

Figure 4. Cumulative distribution of the number of $X$-ray sources as a function of the number of source X-ray counts discovered in the GBS for four different quadrants according to the Galactic coordinates of the sources $(-l,-b)$, $(-l,+b),(+l,+b)$, and $(+l,-b)$. The full histogram shows the cumulative difference in the number of $\mathrm{X}$-ray sources as a function of the number of detected source X-rays found in the $(+l,-b)$ and the $(-l,-b)$ quadrants. The difference is qualitatively the same when comparing the number of X-ray sources in $(+l,-b)$ to the numbers in the other quadrants. There is a clear excess of number of $\mathrm{X}$-ray sources discovered in the $(+l,-b)$ quadrant when compared with the other quadrants. The difference increases with X-ray count rate up to sources with $\lesssim 10$ X-ray counts per source.

(A color version of this figure is available in the online journal.)

source and we counted the number of sources in each quadrant $(-l,-b: \# 382),(-l,+b: \# 399),(+l,+b: \# 366)$ and $(+l,-b$ : \#493). It turns out that the quadrant $(+l,-b)$ is responsible for the apparent asymmetry in the number of detected sources (see Figure 4).

Most of the sources we expected to detect are relatively nearby (within $3 \mathrm{kpc}$; Jonker et al. 2011), nevertheless, the different average extinction in the GBS areas in the four quadrants could

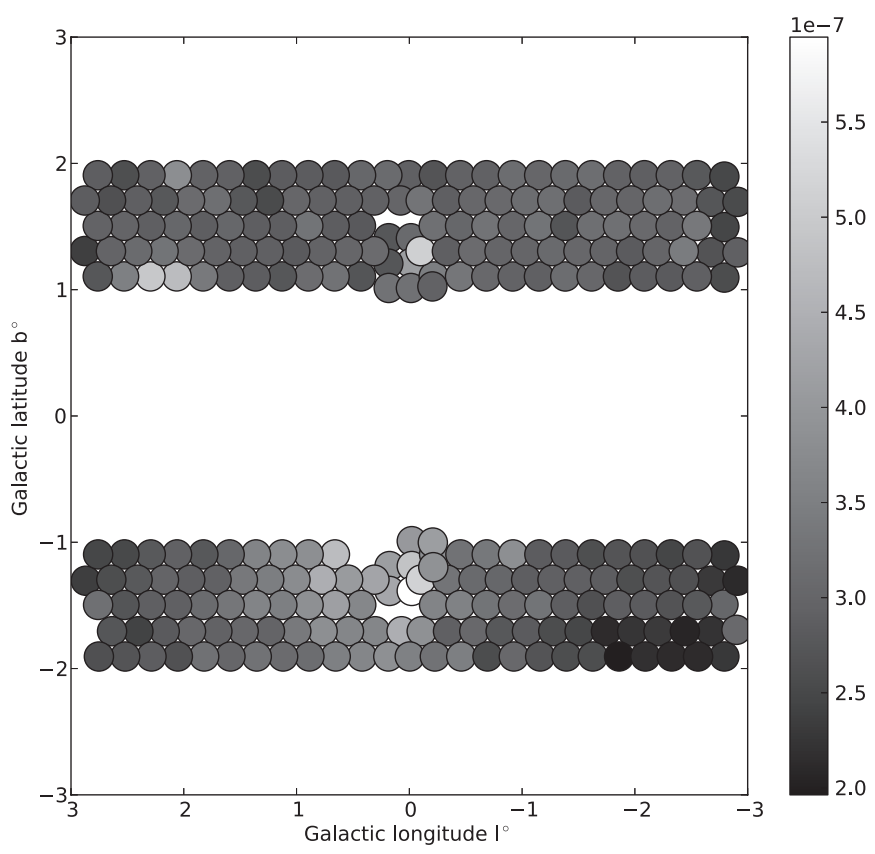

Figure 5. Background count rate (pixel ${ }^{-1} \mathrm{~s}^{-1}$ ) as measured by Chandra. The background is higher in the $(+l,-b)$ part of the GBS area than in the other areas. We removed two observations from this plot-one where we used the FAINT event mode which does not allow for the thorough cleaning of cosmicray afterglow events and therefore yields a much higher background and one where the background is artificially increased due to the presence of a very bright X-ray source.

still have a significant influence on the number of detected sources. The average extinction is indeed lower in the $(+l,-b)$ quadrant where we detected most of the new X-ray sources (see the right panel of Figure 1). The overdensity of sources we find in quadrant $(+l,-b)$ of the GBS area coincides with the presence of diffuse X-ray-emitting gas in that part of the GBS area, as found by ROSAT (Snowden et al. 1997).

In order to investigate this asymmetry further we compared the different background levels in our Chandra observations as determined by the WAVDETECT tool (see Figure 5; a higher background is indicated by a lighter shade of gray). The background levels could influence the detection probability especially for sources with three counts falling far away from the optical axis of the satellite. The diffuse emission could show up as a diffuse number of pixels with one or two counts or in areas with a lower extinction a larger amount of one- and twocount sources such as RS CVn, and coronally active stars might be present.

For a background count rate per pixel per second of $\approx 5 \times 10^{-7}$ (see Figure 5 ) and $2 \mathrm{ks}$ exposures and $\lesssim 100$ pixels for the pointspread function far off-axis, the expected background rate is $\lesssim 0.1$ count per 2 ks observation in such an area. Whereas there is indeed a difference in the background count rate in line with the expectation from either more one- to two-count point sources or more diffuse emission in the $(+l,-b)$ quadrant of the GBS area, this enhanced background does not have a large effect on the number of three-count sources even far off-axis.

We conclude that the overdensity of sources in the $(-l,+b)$ part of our GBS area is likely caused by the lower average extinction in that quadrant of the GBS survey area, whereas the higher X-ray background in that area is in line with the diffuse gas as found by Snowden et al. (1997). Those authors argued that this diffuse gas is at the distance of the bulge. 


\subsection{Comparison with ROSAT Sources}

In order to investigate whether sources in our CXB source list are detected by ROSAT we cross-correlated the GBS CXB source list with the ROSAT All Sky Survey (RASS; Voges et al. 1999). We queried both the Bright as well as the Faint catalog, the ROSAT High Resolution Imager (HRI) Pointed Observations (1RXH), and the Second ROSAT Position Sensitive Proportional Counter (PSPC) Catalog (2RXP) using the VizieR database. To accommodate the relatively large positional uncertainties in many of the ROSAT source detections, we searched for ROSAT sources within $30^{\prime \prime}$ of the Chandra positions of our CXB sources.

We find two RASS (faint) sources that have a position relatively close to the GBS CXB sources CXB9 and CXB11. These sources are probably associated with the ROSAT sources 1RXS J174916.5-311509 and 1RXS J175019.0-302654, respectively. CXB9 is 9"3 away from 1RXS J174916.5-311509. CXB9 is also associated with an O8 III Tycho-2 source (Hynes et al. 2012; see their work for further details on this source). CXB11 is 22" away from 1RXS J175019.0-302654 which is probably the same source as 2RXP J175020.0-302616. We furthermore find that CXB55 likely corresponds to $1 \mathrm{RXH}$ J175017.6-311427 (reported in Rappaport et al. 1994). The angular distance between the two sources is 12 "' 2 . Finally, CXB93

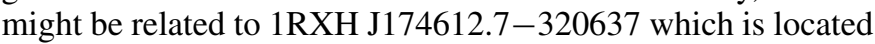
at an angular separation of $25^{\prime \prime}$.

\subsection{Transient Sources}

The first three $\mathrm{CXB}$ sources (CXB1-3) are bright enough that they should have been detected in RASS if they were as bright during RASS as they were in our Chandra observations. However, they were not detected in RASS, and thus we are inclined to conclude that their X-ray luminosity has significantly varied between our Chandra and the RASS observation. Before we could firmly conclude that these sources are variable, we verified the Chandra X-ray spectrum of each of these sources. CXB1 and CXB2 have spectra that should have allowed for a detection in RASS, however, we found that the spectrum of CXB3 is strongly absorbed, potentially providing an explanation as to why ROSAT did not detect the source. Using $C$-statistics we fit a spectral model consisting of a power law absorbed by interstellar material to the X-ray spectrum. For CXB3 we find a best fit $N_{\mathrm{H}}=(2.7 \pm 0.9) \times 10^{22} \mathrm{~cm}^{-2}$ for a power-law index of $2.4 \pm 0.7$. Extrapolating this model to the ROSAT band $(0.01-2.5 \mathrm{keV})$ we find that the source flux

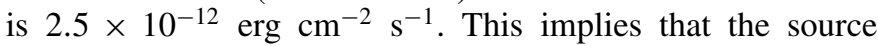
should have been detected by RASS although we note that the extrapolation to low energies carries a significant uncertainty. We tentatively conclude that CXB1, 2, and 3 are transient or at least highly variable sources.

CXB3 has a bright NIR counterpart at $K=10.06 \pm 0.04$ (2MASS J17461440-3219494; this 2MASS source was not picked up in our cross-correlation with SIMBAD; see Section 4) at an angular distance of 0.13 , which is consistent with the $95 \%$ confidence uncertainty on the position of the source of 0 '.31 (see Table 1). The extinction toward the source as given by Gonzalez et al. (2012) is $E(B-V) \sim 2.8$. This yields an $N_{\mathrm{H}} \sim 1.6 \times 10^{22} \mathrm{~cm}^{-2}$ which is consistent within the uncertainties with the value we find from our fit to the X-ray spectrum (using the conversion of $E(B-V)$ to $A_{V}$ using a gas to dust ratio of $R=3.1$ and the conversion from $A_{V}$ to $N_{\mathrm{H}}$ from Predehl \& Schmitt 1995). This value for the extinction is also consistent with a distance to the source of $\sim 8 \mathrm{kpc}$. For that
Table 2

RASS Faint Sources without CXB X-Ray Counterparts within 30"

\begin{tabular}{lcccr}
\hline \hline 1RXS & R.A. & Decl. & $\begin{array}{c}\Delta \\
\left({ }^{\prime \prime}\right)\end{array}$ & $L$ \\
\hline J175237.6-294714 a & 268.1567 & -29.78722 & 49 & 9 \\
J175343.3-291444 & 268.4304 & -29.2457 & 16 & 8 \\
J175342.4-290809 & 268.4267 & -29.1358 & 19 & 10 \\
J175420.8-285412 & 268.5867 & -28.9033 & 15 & 12 \\
J175606.4-283311 & 269.0267 & -28.5532 & 30 & 8 \\
J175712.8-280510 & 269.3033 & -28.0863 & 17 & 10 \\
J175836.1-273358 & 269.6504 & -27.5661 & 19 & 8 \\
J175050.7-301735 & 267.7112 & -30.2932 & 37 & 7 \\
J175323.2-295649 & 268.3467 & -29.9471 & 19 & 8 \\
J175334.9-295013 & 268.3954 & -29.8369 & 14 & 8 \\
J175421.9-292206 & 268.5913 & -29.3683 & 14 & 15 \\
J175855.9-272945 & 269.7329 & -27.4960 & 27 & 9 \\
J175019.0-304843 & 267.5792 & -30.8119 & 30 & 10 \\
J174906.7-311915 & 267.2779 & -31.3208 & 21 & 11 \\
J174608.8-320544 $^{\text {a }}$ & 266.5367 & -32.0956 & 25 & 17 \\
\hline
\end{tabular}

Notes. $\Delta$ is the uncertainty on the source position provided by the RASS. $L$ is the likelihood of source detection $L=-\ln (1-P)$, where $P$ is the probability that the source is real. Those sources with $L \gtrsim 9$ that went undetected in the GBS are good candidate transients.

${ }^{\text {a }}$ Marked in the RASS as a potentially extended ROSAT source.

distance the source luminosity will be around $6 \times 10^{33} \mathrm{erg} \mathrm{s}^{-1}$. The source is also detected in the 2MASS, Wide-field Infrared Survey Explorer (WISE), and GLIMPSE surveys (Skrutskie et al. 2006; Wright et al. 2010; Benjamin et al. 2003; Churchwell et al. 2009 , respectively) as well as in our Blanco/DECam $r^{\prime}$ data at $r^{\prime} \sim 19.8$ (C. B. Johnson et al., in preparation). Correcting for the reddening of Gonzalez et al. (2012) we find that the spectral energy distribution fits well with the Kurucz model (Castelli $\&$ Kurucz 2003) of a late-K red giant of $T_{\text {eff }}=4000 \mathrm{~K}$ and $\log g=1.5$. This source is a candidate symbiotic X-ray binary (see Hynes et al. 2014).

We also investigated whether ROSAT sources found using RASS (Bright and Faint catalogs) as well as pointed observations (from the HRI and the PSPC) fell in the observed GBS CXB area but were not detected. We used TopCAT to crosscorrelate the VizieR ROSAT Catalogues mentioned above with the coordinates of the Chandra pointing centers. We considered a sky area of $7^{\prime}$ around the Chandra pointing centers in this cross-correlation. The resulting list contains all the ROSAT sources that fall inside this sky area. We remove the ROSAT sources that have an associated GBS CXB counterpart within 30 " (see above). Below we discuss the ROSAT sources that were no longer detected in the GBS CXB observations.

1RXH J174423.1-320254 and 1RXH J174449.9-321701 have no CXB counterpart within $30^{\prime \prime}$, however, both sources were detected by ROSAT at signal-to-noise ratios of only 3 and 2.7, respectively. The Second ROSAT PSPC Catalog source 2RXP J175138.6-295024 also went undetected in the CXB. The false alarm probability for the ROSAT detection of this source is $1.2 \times 10^{-2}$.

There are 15 sources from the RASS Faint source catalog within $7^{\prime}$ of a Chandra $\mathrm{CXB}$ pointing that do not have a CXB counterpart within 30" (see Table 2). However, we note that the uncertainty on the position of the faint RASS sources ranges between $14^{\prime \prime}-49^{\prime \prime}$ and the search radius of $30^{\prime \prime}$ might be too strict. However, enlarging the matching radius provides other problems. For example, for a search radius of $1^{\prime}$, 1RXS J174608.8-320544 has two potential CXB counterparts: 
Table 3

NVSS Sources Close to CXB X-Ray Sources

\begin{tabular}{|c|c|c|c|c|c|c|c|}
\hline CXB\# & NVSS & R.A. (J2000) & Decl. (J2000) & $\begin{array}{c}\Delta \text { R.A. } \\
\text { (s) }\end{array}$ & $\begin{array}{c}\Delta \text { Decl. } \\
\left(^{\prime \prime}\right)\end{array}$ & $\begin{array}{l}\mathrm{S} 1.4 \\
(\mathrm{mJy})\end{array}$ & $\begin{array}{c}\text { Separation } \\
\left({ }^{\prime \prime}\right)\end{array}$ \\
\hline CXB19 & $175737-281000$ & 175737.72 & -281000.7 & 0.38 & 9.4 & $3.8 \pm 0.6$ & 7.9 \\
\hline CXB23 & $175230-300107$ & 175230.97 & -300107.8 & 0.03 & 0.6 & $350 \pm 10$ & 0.95 \\
\hline CXB28 & $175205-303026$ & 175205.68 & -303026.7 & 0.43 & 6.7 & $2.8 \pm 0.5$ & 3.6 \\
\hline CXB127 & $174748-312315$ & 174748.62 & -312315.2 & 0.04 & 0.6 & $540 \pm 15$ & 5.0 \\
\hline CXB150 & $175233-295645$ & 175233.16 & -295645.5 & 0.03 & 0.6 & $235 \pm 10$ & 0.71 \\
\hline CXB162 & $173357-302729$ & 173357.85 & -302729.2 & 0.14 & 1.9 & $8.3 \pm 0.5$ & 1.37 \\
\hline CXB163 & $173229-302522$ & 173229.34 & -302522.7 & 0.62 & 8.6 & $2.4 \pm 0.6$ & 17.9 \\
\hline CXB288 & $173251-302919$ & 173251.74 & -302919.4 & 0.05 & 0.7 & $35 \pm 1.2$ & 1.1 \\
\hline CXB384 & $174857-310445$ & 174857.10 & -310445.4 & 0.46 & 8.3 & $5.5 \pm 0.7$ & 3.5 \\
\hline
\end{tabular}

CXB93 and CXB406. CXB93 is at 57.'2 and CXB406 lies at 51".6 from 1RXS J174608.8-320544 (CXB93 and CXB406 are 94 .'7 apart and they are thus not consistent with being the same source). Interestingly, given that 1RXS J174608.8-320544 is marked as a potentially extended source in the RASS catalog, it might be that the source is a blend of CXB93 and CXB406.

For all the sources with $L \gtrsim 9$ in Table 2 as well as the two 1RXH sources and the one 2RXP source not detected in $\mathrm{CXB}$, it is conceivable that the ROSAT observations found the source in a bright state and/or that the source spectrum is too soft to allow for a detection in the GBS CXB observations. Several sources present secure ROSAT detections and they should have been detected in our CXB observations, e.g., 1RXS J175421.9-292206 is detected at more than $5 \sigma$ significance with ROSAT hardness ratio $1(\mathrm{HR} 1)=0.43 \pm 0.37$ and hardness ratio $2(\mathrm{HR} 2)=0.22 \pm 0.42$. Here, $\mathrm{HR} 1=(B-A) /(B+A)$ and HR2 $=(D-C) /(D+C)$, with $A=0.11-0.41 \mathrm{keV}, B=$ $0.52-2.0 \mathrm{keV}, C=0.5-0.9 \mathrm{keV}$, and $D=0.9-2.0 \mathrm{keV}$ count rate. Therefore, the X-ray spectrum is not too soft for Chandra, indicating that this source has varied between the ROSAT and the Chandra observations. For some other sources, most notably those with $L \lesssim 9$ in Table 2 , the ROSAT detection significance is also so low that they could be spurious detections.

\section{RADIO NVSS DETECTIONS AND SIMBAD LISTING OF GBS CXB SOURCES}

After Maccarone et al. (2012) we provide the result from the cross-correlation between the CXB source list and the NRAO VLA Sky Survey (NVSS), where NRAO and VLA stand for National Radio Astronomy Observatory and Very Large Array, respectively. We considered sources within $30^{\prime \prime}$ of a CXB source as a likely match. Table 3 contains the nine NVSS sources we find and their likely CXB counterparts.

The three radio-bright objects associated with CXB23, CXB127, and CXB150 are also detected in Nord et al. (2004) as $330 \mathrm{MHz}$ sources called GCPS 359.845-1.845 $\left(\Delta=3^{\prime \prime} .8\right.$; $\left.S_{330 \mathrm{MHz}}=764 \mathrm{mJy}\right)$, GCPS 358.154-1.680 $\left(\Delta=17^{\prime \prime}\right.$; $\left.S_{330 \mathrm{MHz}}=1464 \mathrm{mJy}\right)$, and GCPS 359.912-1.815 ( $\Delta=3^{\prime \prime} .6$; $\left.S_{330 \mathrm{MHz}}=474 \mathrm{mJy}\right)$, respectively. For $S_{v} \propto v^{\alpha}$, where $v$ is the radio frequency and $S_{v}$ is the radio flux, this yields $\alpha=-0.5,-0.7,-0.5$, respectively. These sources have radio spectra consistent with being AGNs and we thus preliminary classify CXB23, CXB127, and CXB150 as such.

For the other GBS CXB sources with potential radio counterparts it is more difficult to provide a classification on the basis of the potential association with the radio source alone.

Finally, we cross-correlated the positions of the CXB sources with the entries in SIMBAD where we retained optical sources that have a position within $5^{\prime \prime}$ of that of a CXB source and radio and X-ray sources that have a position within $30^{\prime \prime}$ from a CXB source. Table 4 contains the resulting list of sources. Some of the NVSS sources are not found this way (compare with Table 3) whereas others are (e.g., the match between the NVSS source and CXB23 is also found using SIMBAD). Many of the associations of CXB sources with bright optical counterparts were already found in Hynes et al. (2012). Note that some CXB sources have multiple entries as they have more than one potential counterpart within $5^{\prime \prime}$, such as CXB93, CXB256, and CXB422, or they have multiple detections of presumably the same object with slightly different positions such CXB9, CXB23, and CXB150.

In order to estimate the number of false positive identifications, we then shifted all the CXB source positions by $15^{\prime \prime}$ or $30^{\prime \prime}$ north or south, and we redid the cross-correlation. On average, we get 5.5 SIMBAD matches and almost all of these spurious matches are Optical Gravitational Lensing Experiment (OGLE) sources, with a few matches to stars from the open cluster NGC 6451 . Thus, we therefore conclude that $\sim 38$ of our 43 optical/IR matches are real matches, with the OGLE matches being subject to the highest false alarm probability.

From Table 4, we find three cataclysmic variables with close positional matches to the CXB X-ray source positions (CXB10, CXB26, and CXB245). These associations are probably all real. Finally, CXB97 is well matched with a W UMa type source. This is likely to be a real match, and part of the predicted W UMa population.

\section{SUMMARY}

In this paper we have presented the Chandra source list and some properties of the X-ray sources of observations covering the $\approx$ quarter of the total survey area of 12 square degrees remaining to be done after the work of Jonker et al. (2011). This paper thus completes the Chandra survey part of the GBS. The accurate Chandra source positions will help identify the optical, NIR, and UV counterparts. The 424 X-ray sources that have been discovered here, together with the 1216 unique sources from Jonker et al. (2011), compare well with the total number of $\approx 1650 \mathrm{X}$-ray sources that we predicted we should detect in the full 12 square degrees. However, this is of course no guarantee that the number of sources per source class is close to the number we calculated. Optical and NIR photometry including variability information and spectroscopy is necessary to determine the nature of each of the sources (see for instance Ratti et al. 2013; Britt et al. 2013; Hynes et al. 2014; Torres et al. 2013). 
Table 4

Optical or Near-infrared Sources Found in the SIMBAD Data Base within 5" of CXB X-ray Sources

\begin{tabular}{|c|c|c|c|c|c|c|c|c|}
\hline CXB\# & CXB\# & R.A. J2000-CXB & Decl. J2000-CXB & Angular D & Simbad Name & R.A. J2000 & Decl. J2000 & ID \\
\hline 1 & CXB2 & 268.499460 & -29.4852781 & 7.2 & AX J1754.0-2929 & 268.500000 & -29.483333 & $\mathrm{X}$ \\
\hline 2 & $\mathrm{CXB5}^{\mathrm{a}}$ & 263.0362304 & -30.474635 & 0.2 & HD 315961 & 263.036154 & -30.474636 & K5 \\
\hline $3 a$ & CXB9 $9^{\mathrm{a}}$ & 267.3192035 & -31.2550666 & 0.6 & HD 161853 & 267.319017 & -31.255022 & O8 III \\
\hline $3 b$ & CXB9 & 267.3192035 & -31.2550666 & 3.4 & PN RPZM 40 & 267.319583 & -31.254167 & $\mathrm{PN} ?$ \\
\hline $3 c$ & СXB9 & 267.3192035 & -31.2550666 & 10 & 1RXS J174916.5-311509 & 267.318671 & -31.252244 & $\mathrm{X}$ \\
\hline 4 & CXB10 & 269.6350093 & -27.8789043 & 0.7 & MACHO 401.48296 .2600 & 269.635208 & -27.879000 & $\mathrm{CV}$ \\
\hline 5 & CXB11 & 267.5862652 & -30.4477944 & 22 & 1RXS J175019.0-302654 & 267.579158 & -30.448469 & $\mathrm{X}$ \\
\hline 6 & $\mathrm{CXB} 17^{\mathrm{a}}$ & 268.6255656 & -29.3992464 & 0.2 & 2MASS J17543011-2923572 & 268.625488 & -29.399244 & IR \\
\hline 7 & CXB21 & 268.7011304 & -29.3277772 & 3.7 & OGLE BUL-SC4 568004 & 268.700458 & -29.328611 & $\mathrm{~V}^{*}$ \\
\hline $8 \mathrm{a}$ & CXB23 & 268.1288255 & -30.0186408 & 0.6 & [IBR2011] J1752-3001 & 268.128960 & -30.018515 & Radio \\
\hline $8 \mathrm{~b}$ & CXB23 & 268.1288255 & -30.0186408 & 1.5 & [LKL2000] 43 & 268.129167 & -30.018333 & Radio \\
\hline $8 \mathrm{c}$ & CXB23 & 268.1288255 & -30.0186408 & 4.3 & GCPS 111 & 268.130208 & -30.018500 & Radio \\
\hline 9 & CXB26 & 268.4491784 & -29.7439772 & 1.0 & OGLE BUL-SC3 6033 & 268.448875 & -29.743861 & $\mathrm{CV}$ \\
\hline 10 & CXB28 & 268.0240465 & -30.5064844 & 2.1 & 2XMM J175205.6-303023 & 268.023375 & -30.506556 & $\mathrm{X}$ \\
\hline 11 & CXB29 & 268.5549195 & -29.4830887 & 0.6 & OGLE BUL-SC4 155897 & 268.554750 & -29.483028 & $\mathrm{~V}^{*}$ \\
\hline 12 & CXB34 & 266.8706341 & -32.2448156 & 12 & 2MASS J17472806-3214462 & 266.866917 & -32.246194 & $\mathrm{X}$ \\
\hline 13 & $\mathrm{CXB} 36^{\mathrm{a}}$ & 266.5600100 & -32.1033654 & 4.1 & LTT 7073 & 266.560160 & -32.102233 & $\mathrm{PM}^{*} \mathrm{M} 2 \mathrm{~V}$ \\
\hline 14 & CXB49 & 267.3703237 & -31.3067944 & 0.8 & 2MASS J17492885-3118237 & 267.370225 & -31.306603 & Candidate YSO \\
\hline 15 & CXB54 & 268.1172224 & -29.9895816 & 13 & RRF 9 & 268.114167 & -29.987222 & Radio \\
\hline 16 & CXB55 & 267.5735447 & -31.2430775 & 12 & [RDL94] Terzan 6 A & 267.574167 & -31.239722 & $\mathrm{X}$ \\
\hline 17 & CXB58 & 268.5832235 & -29.6379212 & 0.8 & 2MASS J17541996-2938157 & 268.583188 & -29.637694 & $\mathrm{~EB}^{*}$ \\
\hline 18 & CXB63 & 267.6738181 & -30.1941350 & 1.3 & $\mathrm{Cl}^{*}$ NGC $6451 \mathrm{KF} 227$ & 267.674208 & -30.194250 & in Cluster \\
\hline $19 \mathrm{a}$ & $\mathrm{CXB}^{\mathrm{a}}{ }^{\mathrm{a}}$ & 266.5529013 & -32.1035349 & 2.5 & LTT 7072 & 266.552088 & -32.103529 & $\mathrm{PM}^{*} \mathrm{M} 2 \mathrm{~V}$ \\
\hline $19 b$ & $\mathrm{CXB93}^{\mathrm{a}}$ & 266.5529013 & -32.1035349 & 3.7 & ** LDS 611/GJ $2130 \mathrm{C}$ & 266.553167 & -32.102528 & $* *$ \\
\hline 20 & CXB97 & 269.7613953 & -27.4890113 & 0.9 & $\mathrm{~V}^{*} \mathrm{~V} 1723 \mathrm{Sgr}$ & 269.761125 & -27.488917 & EB*WUMa \\
\hline 21 & CXB100 & 268.4645298 & -29.650292 & 2.3 & OGLE BUL-SC3 769186 & 268.464292 & -29.650889 & $\mathrm{~V}^{*}$ \\
\hline 22 & CXB112 & 263.2739071 & -30.5863552 & 2.0 & LP $920-61$ & 263.274083 & -30.585833 & $\mathrm{PM}^{*} \mathrm{M} 2.5$ \\
\hline 23 & $\mathrm{CXB} 116^{\mathrm{a}}$ & 269.2814150 & -27.1476849 & 0.4 & HD 314886 & 269.281369 & -27.147590 & A5 \\
\hline 24 & CXB127 & 266.9509625 & -31.3875612 & 3.0 & NVSS J174748-312315 & 266.950958 & -31.388389 & Radio \\
\hline 25 & $\mathrm{CXB} 128^{\mathrm{a}}$ & 266.7138646 & -25.7794799 & 1.5 & CD-25 12283 & 266.714287 & -25.779338 & F8 \\
\hline $26 a$ & CXB 150 & 268.1381712 & -29.9457729 & 0.7 & VCS4 J1752-2956 & 268.137946 & -29.945806 & Radio \\
\hline $26 b$ & CXB150 & 268.1381712 & -29.9457729 & 3.5 & GCPS 115 & 268.139292 & -29.945750 & Radio \\
\hline 27 & $\mathrm{CXB} 181^{\mathrm{a}}$ & 268.73059000 & -29.2027756 & 0.3 & HD 162962 & 268.730569 & -29.202854 & A \\
\hline 28 & CXB183 & 268.6757225 & -28.8307272 & 3.0 & IRAS $17515-2849$ & 268.674792 & -28.830500 & Star \\
\hline 29 & CXB200 & 263.4644661 & -30.8417862 & 0.5 & TYC 7376-433-1 & 263.464475 & -30.841914 & Star \\
\hline 30 & $\mathrm{CXB} 211^{\mathrm{a}}$ & 265.8693744 & -32.2325220 & 2.6 & HD 160826 & 265.870188 & -32.232264 & B9 V \\
\hline 31 & $\mathrm{CXB} 225^{\mathrm{a}}$ & 269.0803986 & -28.4701699 & 2.5 & TYC 6853-3032-1 & 269.079825 & -28.470642 & Star \\
\hline 32 & $\mathrm{CXB} 233^{\mathrm{a}}$ & 268.83897484 & -28.5734201 & 1.1 & HD 316692 & 268.839115 & -28.573143 & $\mathrm{~A} 0$ \\
\hline 33 & CXB245 & 268.2919765 & -29.3556874 & 0.5 & OGLE J175310.04-292120.6 & 268.291833 & -29.355722 & Dwarf Nova \\
\hline $34 \mathrm{a}$ & CXB256 & 267.7514663 & -30.3199539 & 1.7 & $\mathrm{Cl}^{*}$ NGC 6451 PMR 65 & 267.751250 & -30.319528 & in Cluster \\
\hline $34 \mathrm{~b}$ & CXB256 & 267.7514663 & -30.3199539 & 1.7 & $\mathrm{Cl}^{*}$ NGC 6451 PMR 64 & 267.751917 & -30.319667 & in Cluster \\
\hline 35 & $\mathrm{CXB} 287^{\mathrm{a}}$ & 263.3901785 & -30.534113 & 1.5 & HD 158982 & 263.389732 & -30.533990 & A2 IV/V \\
\hline 36 & CXB293 & 268.710370 & -29.3371961 & 0.4 & 2MASS J17545048-2920142 & 268.710375 & -29.337306 & $\mathrm{~EB}^{*}$ \\
\hline 37 & $\mathrm{CXB} 302^{\mathrm{a}}$ & 269.6706800 & -27.9024008 & 0.3 & TYC 6849-1627-1 & 269.670621 & -27.902478 & Star \\
\hline 38 & $\mathrm{CXB}_{306}^{\mathrm{a}}$ & 269.5399321 & -28.1418302 & 0.4 & HD 163613 & 269.539931 & -28.141712 & B1 Iab \\
\hline 39 & CXB352 & 268.4262642 & -29.8320194 & 1.3 & OGLEII DIA BUL-SC3 5152 & 268.426333 & -29.831667 & $\mathrm{~EB}^{*}$ \\
\hline 40 & CXB361 & 268.1649063 & -29.752345 & 5.0 & OGLE BUL-SC37 441760 & 268.163375 & -29.751944 & $\mathrm{~V}^{*}$ \\
\hline 41 & CXB366 & 268.1003203 & -29.7169994 & 0.2 & 2MASS J17522407-2943013 & 268.100292 & -29.717056 & $\mathrm{~EB}^{*}$ \\
\hline 42 & CXB380 & 267.3212976 & -31.2837757 & 11.5 & SNR G358.4-01.9 & 267.325000 & -31.283333 & SNR \\
\hline $43 \mathrm{a}$ & $\mathrm{CXB} 422^{\mathrm{a}}$ & 262.8208422 & -30.3215429 & 0.8 & HD 315956 & 262.820644 & -30.321404 & F2 \\
\hline $43 b$ & $\mathrm{CXB} 422^{\mathrm{a}}$ & 262.8208422 & -30.3215429 & 3.2 & [RHI84] 9-186 & 262.820125 & -30.320917 & M4 \\
\hline
\end{tabular}

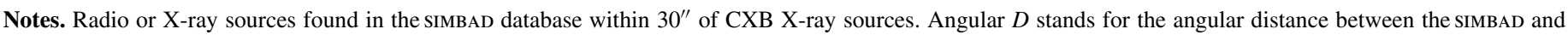

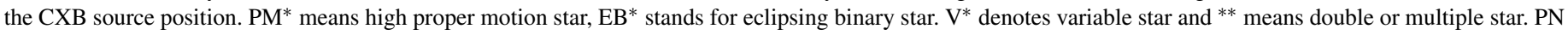
stands for planetary nebula and YSO for young stellar object. Finally, supernova remnant is abreviated as SNR and cataclysmic variable as CV.

a Association already found in Hynes et al. (2012).

We discussed the apparent overdensity of sources in the $(+l,-b)$ quadrant of the GBS area. We conclude that this is caused by the lower extinction in this quadrant.

We compared our source list with that of RASS. Furthermore, we compared our Chandra source list with the sources found in the catalog of sources derived from pointed HRI and PSPC ROSAT observations that fall inside the GBS area. Finally, we investigate whether some of the sources we report on here are present in public radio surveys.

The authors thank the CXC for scheduling the Chandra observations. R.I.H., C.T.B., and C.B.J., acknowledge support from the National Science Foundation under grant No. AST0908789. This research has made use of the SIMBAD database, 
operated at CDS, Strasbourg, France. This research has made use of the VizieR Catalogue access tool, CDS, Strasbourg, France. The original description of the VizieR service was published in A\&AS, 143, 23. This publication makes use of data products from the Wide-field Infrared Survey Explorer, which is a joint project of the University of California, Los Angeles, and the Jet Propulsion Laboratory/California Institute of Technology, funded by the National Aeronautics and Space Administration (NASA). This work is based in part on observations made with the Spitzer Space Telescope, which is operated by the Jet Propulsion Laboratory, California Institute of Technology under a contract with NASA.

\section{REFERENCES}

Arnaud, K. A. 1996, in ASP Conf. Ser. 101, Astronomical Data Analysis Software and Systems V, ed. G. H. Jacoby \& J. Barnes (San Francisco, CA: ASP), 17

Belczynski, K., \& Taam, R. E. 2004, ApJ, 603, 690

Belczynski, K., Wiktorowicz, G., Fryer, C. L., Holz, D. E., \& Kalogera, V. 2012, ApJ, 757, 91

Benjamin, R. A., Churchwell, E., Babler, Brian L., et al. 2003, PASP, 115, 953 Britt, C. T., Torres, M. A. P., Hynes, R. I., et al. 2013, ApJ, 769, 120

Castelli, F., \& Kurucz, R. L. 2003, in Modelling of Stellar Atmospheres, New Grids of ATLAS9 Model Atmospheres, ed. N. Piskunov et al. (IAU Symposium, Vol. 210; San Francisco, CA: ASP), A20

Churchwell, E., Babler, B. L., Meade, M. R., et al. 2009, PASP, 121, 213

Evans, I. N., Primini, F. A., Glotfelty, K. J., et al. 2010, ApJS, 189, 37

Farr, W. M., Sravan, N., Cantrell, A., et al. 2011, ApJ, 741, 103

Fryer, C. L., Belczynski, K., Wiktorowicz, G., et al. 2012, ApJ, 749, 91

Garmire, G. P. 1997, BAAS, 29, 823

Gehrels, N. 1986, ApJ, 303, 336

Gonzalez, O. A., Rejkuba, M., Zoccali, M., et al. 2012, A\&A, 543, A13

Greiss, S., Steeghs, D., Jonker, P. G., et al. 2014, MNRAS, in press (arXiv:1312.2958)
Grimm, H., McDowell, J., Zezas, A., Kim, D., \& Fabbiano, G. 2005, ApJS, 161,271

Hong, J. S., van den Berg, M., Grindlay, J. E., \& Laycock, S. 2009, ApJ, 706, 223

Hynes, R. I., Wright, N. J., Maccarone, T. J., et al. 2012, ApJ, 761, 162

Hynes, R. I., Torres, M. A. P., Heinke, C. O., et al. 2014, ApJ, 780, 11

Ivanova, N., Justham, S., Chen, X., et al. 2013, A\&ARv, 21, 59

Jonker, P. G., Bassa, C. G., Nelemans, G., et al. 2011, ApJS, 194, 18

Kiel, P. D., \& Hurley, J. R. 2006, MNRAS, 369, 1152

Kim, D., Cameron, R. A., Drake, J. J., et al. 2004, ApJS, 150, 19

King, A. R., \& Ritter, H. 1999, MNRAS, 309, 253

Kreidberg, L., Bailyn, C. D., Farr, W. M., \& Kalogera, V. 2012, ApJ, 757, 36

Lasota, J.-P. 2008, NewAR, 51, 752

Lee, C.-H., Brown, G. E., \& Wijers, R. A. M. J. 2002, ApJ, 575, 996

Maccarone, T. J., Torres, M. A. P., Britt, C. T., et al. 2012, MNRAS, 426, 3057

Murray, S. S., Kenter, A., Forman, W. R., et al. 2005, ApJS, 161, 1

Narayan, R., \& McClintock, J. E. 2005, ApJ, 623, 1017

Nord, M. E., Lazio, T. J. W., Kassim, N. E., et al. 2004, AJ, 128, 1646

Özel, F., Psaltis, D., Narayan, R., \& McClintock, J. E. 2010, ApJ, 725, 1918

Özel, F., Psaltis, D., Narayan, R., \& Santos Villarreal, A. 2012, ApJ, 757, 55

Pfahl, E., Rappaport, S., \& Podsiadlowski, P. 2002, ApJL, 571, L37

Predehl, P., \& Schmitt, J. H. M. M. 1995, A\&A, 293, 889

Rappaport, S., Dewey, D., Levine, A., \& Macri, L. 1994, ApJ, 423, 633

Ratti, E. M., van Grunsven, T. F. J., Jonker, P. G., et al. 2013, MNRAS, 428,3543

Schlegel, D. J., Finkbeiner, D. P., \& Davis, M. 1998, ApJ, 500, 525

Shahbaz, T., \& Kuulkers, E. 1998, MNRAS, 295, L1

Skrutskie, M. F., Cutri, R. M., Stiening, R., et al. 2006, AJ, 131, 1163

Snowden, S. L., Egger, R., Freyberg, M. J., et al. 1997, ApJ, 485, 125

Torres, M. A. P., Jonker, P. G., Britt, C. T., et al. 2013, arXiv:1310.0224

Udalski, A., Kowalczyk, K., Soszynski, I., et al. 2012, AcA, 62, 133

Voges, W., Aschenbach, B., Boller, Th., et al. 1999, A\&A, 349, 389

Wang, Q. D., Gotthelf, E. V., \& Lang, C. C. 2002, Natur, 415, 148

Warwick, R. S., Pérez-Ramírez, D., \& Byckling, K. 2011, MNRAS, 413, 595

Weisskopf, M. C., Brinkman, B., Canizares, C., et al. 2002, PASP, 114, 1

Wright, E. L., Eisenhardt, P. R. M., Mainzer, A. K., et al. 2010, AJ, 140, 1868

Wu, Y. X., Yu, W., Li, T. P., Maccarone, T. J., \& Li, X. D. 2010, ApJ, 718,620 